\title{
Direct observation of mode-coupling instability in two-dimensional plasma crystals
}

\author{
L. Couëdel, * V V. Nosenko, A. V. Ivlev, S. K. Zhdanov, H. M. Thomas, and G. E. Morfill \\ Max Planck Institute for Extraterrestrial Physics, 85741 Garching, Germany
}

\begin{abstract}
Dedicated experiments on melting of $2 \mathrm{D}$ plasma crystals were carried out. The melting was always accompanied by spontaneous growth of the particle kinetic energy, suggesting a universal plasma-driven mechanism underlying the process. By measuring three principal dust-lattice (DL) wave modes simultaneously, it is unambiguously demonstrated that the melting occurs due to the resonance coupling between two of the DL modes. The variation of the wave modes with the experimental conditions, including the emergence of the resonant (hybrid) branch, reveals exceptionally good agreement with the theory of mode-coupling instability.
\end{abstract}

PACS numbers: 52.27.Lw, 52.27.Gr

Strongly coupled complex (dusty) plasmas play an important role in the existing hierarchy of soft matter states 1]. Along with complex fluids and granular media as prominent examples of "regular" soft matter, complex plasmas represent natural model systems which enable remarkably broad interdisciplinary research. The characteristic length- and timescales in such systems are dramatically stretched (e.g., in complex plasmas - to hundreds of microns and tens of milliseconds, respectively). Therefore, numerous generic processes occurring in classical fluids or solids can be studied in greatest detail, at the most fundamental "atomistic" level [1-3] . Especially important here are $2 \mathrm{D}$ systems, where the complete information about all particles can be obtained at each moment of time. The current experimental capabilities make 2D complex plasmas ideal for comprehensive experimental studies of the atomistic dynamics [1, 2, 4, 5 ].

The investigation of atomistic processes that trigger the melting and crystallization is of particular interest. It is well known that the mechanisms resulting in the destruction of the long-range crystalline order in $2 \mathrm{D}$ systems can be very different from those operating in 3D crystals: The classical Kosterlitz-Thouless-HalperinNelson-Young (KTHNY) theory of 2D melting [6] predicts two consecutive phase transitions (with an intermediate, so called "hexatic phase" in between). The alternative theory [7] relates 2D melting with the formation of dislocation chains ("grain boundaries") percolating the system. These are generic melting mechanisms which can operate in very different strongly coupled systems [8 12].

When studying generic classical phenomena occurring in regular liquids and solids, the relevance of a model system (be it colloidal suspensions, granular media, or complex plasmas) becomes crucial. Of course, careful analysis is required in the context of a given phenomenon (or, class of phenomena), but one can certainly identify essential common principles. In particular, the applicability of the Hamiltonial approach for the analysis of atomistic dynamics (for instance, to investigate kinetics of phase transitions) is one of these basic principles. This implies that non-Hamiltonian (non-conservative) behavior peculiar to model systems should play minor role at the relevant timescales $[13]$.

Non-Hamiltonial behavior of particle ensembles is often a manifestation of asymmetry, or nonreciprocity, in the interparticle interactions. Such asymmetry can be considered as a result of the system openness: In the presence of strong flow of the ambient plasma the screening cloud around each charged particle becomes highly asymmetric. These clouds, which are usually referred to as "plasma wakes" [14, 15], play the role of an (external) "third body" in the interparticle interaction and hence make it nonreciprocal [16, 17]. One should point out that such nonreciprocity is quite universal and can be very important for the hydrodynamic interactions of colloids [18] or droplets in conventional fluid flows [19].

The nonreciprocity of pair interaction in complex plasmas provides a very efficient mechanism of converting the energy of the flowing ions into the kinetic energy of microparticles [1, 2, 20]. In particular, this can be associated with dust-lattice (DL) wave modes [2, 21, 22]: The kinetic energy can grow due to resonant particle-wake interactions when different oscillation modes of interacting particles are properly synchronized. Ivlev and Morfill 23] suggested that the resonance between DL wave modes in a 2D plasma crystal can trigger the mode-coupling instability which, in turn, can cause the crystal melting. By comparing the melting experiments with the molecular dynamic simulations, this hypothesis has received strong confirmation [24]. However, the direct proof that the crystalline monolayers can indeed melt due to the mode-coupling instability (and even that the instability itself can be observed in experiments) was still outstanding. One should point out that the other known plasmarelated scenarios of $2 \mathrm{D}$ crystal melting either require the presence of localized defects - single particles (or even sublayers) above or below the monolayer 25], or they are associated with the charge fluctuations and can only operate at very low pressures (well below $1 \mathrm{~Pa}$ ) 26, 27].

In this Letter we report on dedicated experimental investigation of $2 \mathrm{D}$ plasma crystal melting. We explored a fairly broad range of experimental parameters chosen well inside the crystalline regime predicted by the generic (KTHNY or grain-boundary) melting scenarios 


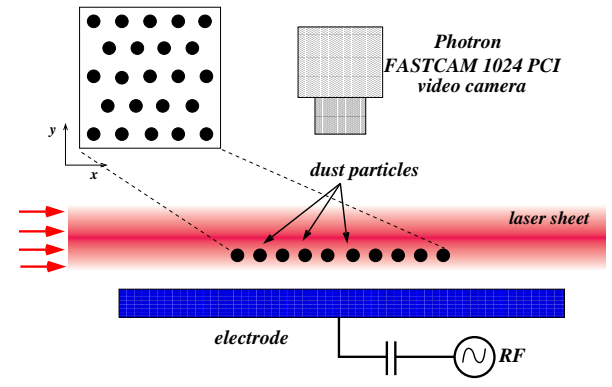

FIG. 1: Sketch of the experimental setup. Microparticles are confined above the RF electrode and are illuminated with a horizontal laser sheet having a Gaussian profile in the vertical direction (with a standard deviation $\sigma=75 \mu \mathrm{m}$ ). The monolayer is levitated well below the peak of the laser intensity, which results in strong intensity variations of the scattered light upon the vertical displacement of individual particles. The upward/downward displacement corresponds to positive/negative intensity variation. Microparticles are recorded from top at a speed of 250 frames per second.

[12]. The melting was always accompanied by a spontaneous growth of the microparticle kinetic energy, which clearly suggests a universal plasma-driven mechanism underlying the process. By implementing simultaneous measurements of the dispersion relations for three principal DL wave modes, we unambiguously demonstrated that the melting occurred according to the mechanism predicted by Ivlev and Morfill. The variation of the DL modes with the experimental conditions, including the emergence of the resonant (hybrid) mode, revealed exceptionally good agreement with the theoretical predictions of the mode-coupling instability.

The melting was systematically investigated in experiments performed with a (modified) GEC chamber, in a capacitively coupled rf glow discharge at $13.56 \mathrm{MHz}$ (see Fig. 1). The argon pressure was between $0.4 \mathrm{~Pa}$ and 1 $\mathrm{Pa}$ and the forward $\mathrm{rf}$ power was between $5 \mathrm{~W}$ and $20 \mathrm{~W}$ (which corresponded to rf peak-to-peak voltage between $175 \mathrm{~V}$ and $310 \mathrm{~V}$ ). A horizontal monolayer was formed by levitating melamine-formaldehyde particles in the plasma sheath above the lower rf electrode. We used particles of two different sizes, $8.77 \pm 0.14 \mu \mathrm{m}$ and $9.15 \pm 0.14 \mu \mathrm{m}$ diameter. A high quality of the monolayer (with no detected particles above or below) was ensured by using a side-view camera. The diameter of the obtained crystalline structure was up to $\simeq 60 \mathrm{~mm}$, depending on the number of injected particles (we explored a range from 1000 to 15,000$)$.

One of two different discharge parameters was used as the control parameter for the melting: We either gradually decreased the rf power at a constant gas pressure (which lowers the resonance frequency of the vertical confinement $f_{\mathrm{v}}$, see Ref. 28]), or we decreased the pressure at a constant $r f$ power (which naturally lowers neutral gas friction, but also makes the radial confinement stronger
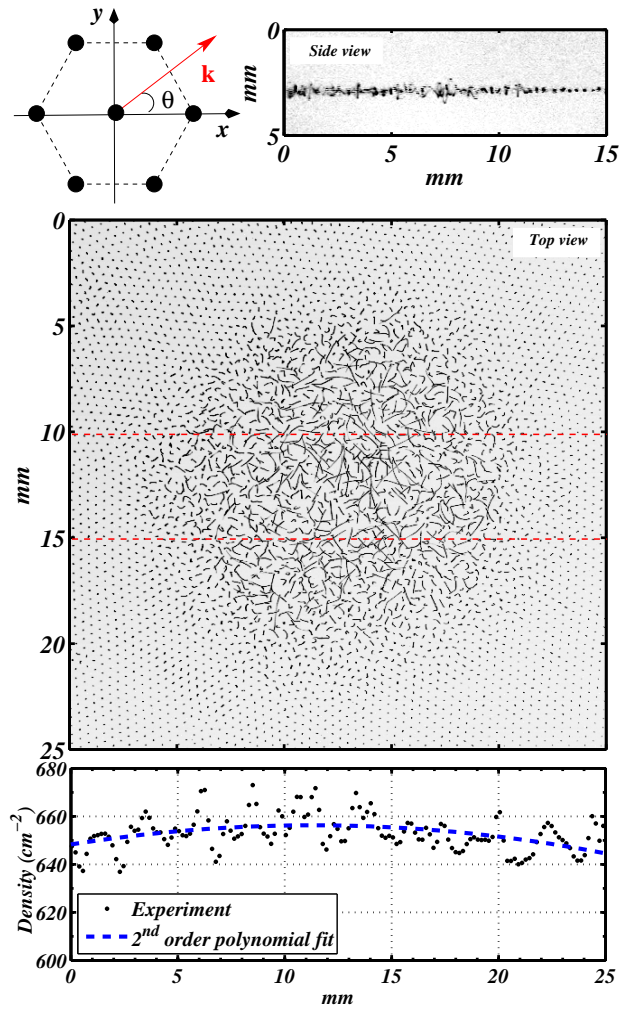

FIG. 2: Example of the instability onset in a 2D plasma crystal. The shown example is for the experiment performed at a rf power of $20 \mathrm{~W}$ with particles of $8.77 \mu \mathrm{m}$ diameter. The pressure of argon was ramped down in steps of $0.02 \mathrm{~Pa}$ (every $\simeq 300 \mathrm{~s}$ ), the instability was triggered at $0.90 \mathrm{~Pa}$. The particle trajectories (superposition of consecutive video frames during $0.04 \mathrm{~s}$ ) are plotted about $50 \mathrm{~s}$ after the critical pressure decrease, the upper and middle panels demonstrate the side and top views, respectively. The lower panel shows the profile of the particle density in the monolayer measured before the instability onset (within the stripe marked in the middle panel by two horizontal dashed lines). In the upper left corner the elementary cell of the hexagonal lattice is depicted with the frame of reference chosen in this paper, due to the lattice symmetry we consider the wave vector $\mathbf{k}$ at $0^{\circ} \leq \theta \leq 30^{\circ}$.

and hence the interparticle distance $a$ smaller) 29]. In either case, the melting developed in the same way, as illustrated in Fig. 2 (see also supplemental movie S1 [30]): Upon decrease of the control parameter below a certain threshold the instability was triggered in the central part of the crystal (i.e., where the particle density was the highest) and then expanded outwards. The instability always set in simultaneously in the horizontal and vertical directions. At the initial stage it was accompanied by an exponential growth of the particle kinetic energy, eventually resulting in the total disruption of the crystal in the unstable zone. Upon increase of the control parameter back above the threshold (usually, with a hysteresis) the system always recrystallized. It is noteworthy that if the rf power was sufficiently high and/or the particle 
density in the monolayer was low enough the crystal remained stable at practically any pressure (e.g., at a rf power of $20 \mathrm{~W}$, particle density of $160 \mathrm{~cm}^{-2}$, and argon pressure down to $p \simeq 0.4 \mathrm{~Pa}$ - the lowest value technically achievable with our setup).

The observed behavior fully agrees with the qualitative picture predicted by the theory of mode-coupling instability [23, 24] : It can only set in when the out-of-plane and in-plane wave modes intersect, which is only possible when $f_{\mathrm{v}}$ is sufficiently low or/and the particle density in the monolayer $\left(\propto a^{-2}\right)$ is high enough. The intersection should be accompanied by the simultaneous excitation of the vertical and horizontal oscillations. On the other hand, the instability should be suppressed by sufficiently high gas friction, i.e., by increasing the gas pressure.

The chief result of our paper is the ultimate proof that the observed melting is indeed triggered by the modecoupling instability. The unique fingerprint characterizing the instability [23, 24] is the crossing of the longitudinal in-plane and transverse out-of-plane DL branches, which results in the (wake-induced) resonance coupling between them and the emergence of an unstable hybrid mode operating in a certain proximity of the crossing. This was directly proved by measuring the dispersion relations of all three principal DL modes. For this purpose, we used the technique by Nunomura et al. 331] of reconstructing the in-plane dispersion relations from thermal fluctuations of particle velocities in the horizontal plane. In addition, we implemented a novel method of particle imaging 22] which allowed us to measure simultaneously the vertical fluctuations and hence to retrieve also the out-of-plane mode. The essential advantage of this technique is that it does not involve any intermediate (model) assumptions about the interparticle interactions, and therefore yields "true" dispersion relations (or "fluctuation spectra") which can be obtained for arbitrary experimental conditions (that just allow the individual particle tracking).

In Fig. 3 we plotted the fluctuation spectra for the out-of-plane mode (upper panel) separately from those for the in-plane modes (second-from-the-top panel), because the imaging method implemented for the simultaneous tracking of the vertical and horizontal fluctuations yields different background intensities 222]. As regards the theoretical curves, in each panel we plotted all principle modes that fit the frequency range shown.

Figure 3 summarizes results of our analysis. In the stable regime (left column) the wave modes are well separated and the fluctuation intensity is evenly distributed over the branches. In contrast, the unstable regime (right column) is characterized by the apparent intersection between the out-of-plane and (one of) in-plane branches. The intersection occurs in the vicinity of the first Brillouin zone boundary, resulting in the anomalous energy release (dark-red "hot spots").

The qualitative effects and features of the mode-

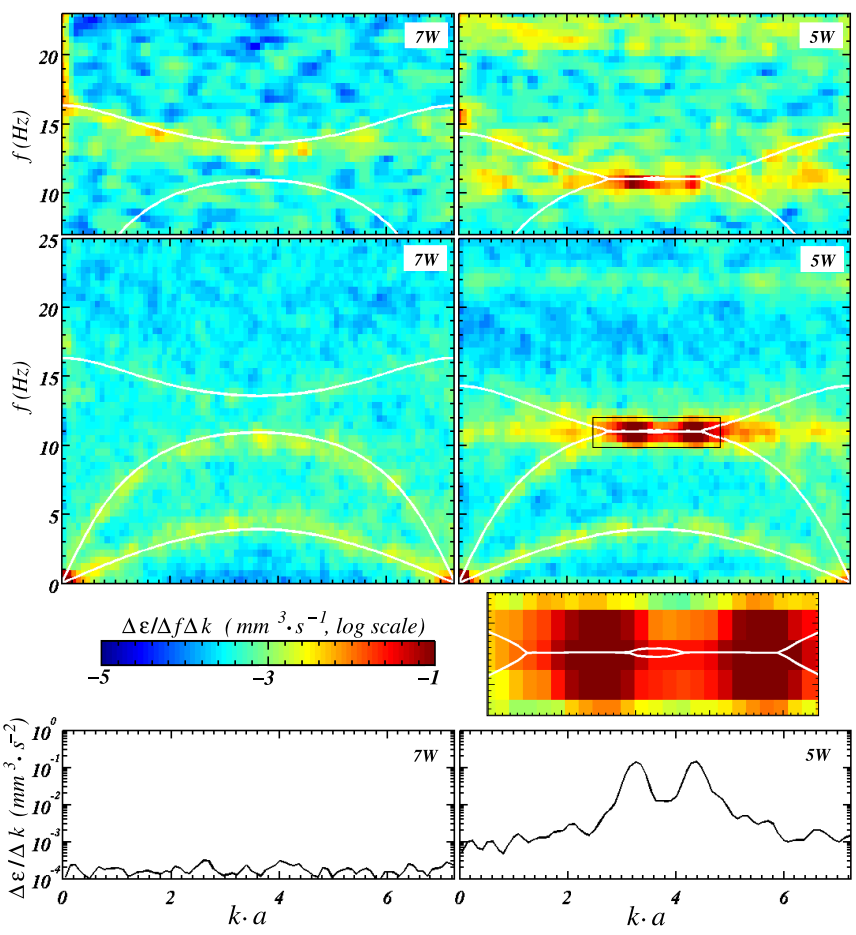

FIG. 3: Evolution of the DL dispersion relations near the instability onset. The dispersions relations ("fluctuation spectra") were measured in the experiment performed at argon pressure of $0.76 \mathrm{~Pa}$ with particles of $9.15 \mu \mathrm{m}$ diameter, at a rf power of $7 \mathrm{~W}$ (left column, stable regime) and $5 \mathrm{~W}$ (right column, the instability onset). The logarithmic color coding is used for the "fluctuation intensity" $\Delta \epsilon / \Delta k \Delta f$ (energy density, for unit mass, per unit wave number and frequency): Yellow ridges represent stable wave modes, the unstable hybrid mode are the dark-red "hot spots"; the upper panel shows the measured out-of-plane mode, the second-from-the-top panel depicts the pair of in-plane modes. The solid lines in both panels are theoretical results [32] (see text for details). The wave number $k$ is normalized by the inverse interparticle distance $a^{-1}$ (determined from the first peak of the pair correlation function; the uncertainty of $a$ was $\simeq 4 \%$ due to density variations, see e.g., the lower panel of Fig. 21). The shown range of $k$ corresponds to the first two Brillouin zones at $\theta=30^{\circ}$ (branches are symmetric with respect to the border between the two zones). Below the fluctuation spectra the magnified hybrid mode in the unstable regime (marked by a rectangular box) is shown. The lower panel illustrates the distribution of the "fluctuation energy" $\Delta \epsilon / \Delta k$ at fixed frequency: In the stable case the frequency $(\simeq 12.5 \mathrm{~Hz})$ is chosen in the middle between the longitudinal in-plane branch and the out-of-plane branch (measured at the border between the Brillouin zones), for the instability onset the frequency $(\simeq 11 \mathrm{~Hz})$ corresponds to the hybrid mode.

coupling instability mentioned above were originally predicted by the theory for a 1D string [23, 24], and they all remain unaltered also in $2 \mathrm{D}$ case. For rigorous quantitative comparison with the experiments we employed the theoretical results for $2 \mathrm{D}$ crystals obtained recently by Zhdanov et al. [32]. The theoretical branches are shown 
in Fig. 3 by solid lines: The upper curve represents the optical out-of-plane mode, the middle and lower curves are for the pair of acoustic in-plane modes (longitudinal and transverse, respectively). These curves were calculated for the normalized dipole moment of the wake $\tilde{q} \tilde{\delta}$ (see Ref. [32]) equal to 0.1. We assumed the Yukawa interparticle interaction, with the parameters (viz., the particle charge and the effective screening length) deduced from the low- $k$ part of in-plane fluctuation spectra (using the method of Ref. [31]).

Figure 3 shows that the theory yields excellent agreement with the experiment, both for the stable and unstable regimes. Especially important is that in the unstable regime (right column) the predicted unstable hybrid mode perfectly coincides with the locations of "hot spots" in the measured fluctuation spectra. Even subtle effects, such as the splitting of "hot spots" at the border between the Brillouin zones is very well resolved (see the distribution of the fluctuation energy in the lower panel, and also the magnified hybrid mode) [33].

The fluctuation spectra shown in Fig. 3 were plotted for the wave vector $\mathbf{k}$ at $\theta=30^{\circ}$ (see Fig. 2), because the theory predicts this orientation to be the most unstable (see Fig. 4 of Ref. [32]). In order to further highlight excellent comprehensive agreement between experiments and theory, we plotted additional spectra for different angles (see supplemental figure S2 30]). They clearly show that at $\theta=30^{\circ}$ the fluctuation intensity of the "hot spots" is indeed the highest.

Note that rapid energy growth after the instability onset results in various nonlinear phenomena which can be easily identified in the right column of Fig. 3. The most prominent effects are the energy cascades from the "hot spots" along the $k$-axis (bright red-yellow stripe in the second-from-the-top panel, see also the energy profile in the lower panel), and the generation of secondary harmonics at the double frequency of the hybrid mode (yellow bands at $\simeq 22 \mathrm{~Hz}$ in the fluctuation spectra).

In conclusion, one should point out that any instability operating in 2D plasma crystals and associated with collective processes [34] should be accompanied by anomalies in the fluctuation spectra. Therefore, the method of simultaneous measurement of all three principal DL modes promises to be very fruitful for further investigation of the melting scenarios. Especially important would be a systematic search for other possible resonant processes that can extend or alter the mechanism reported in this Letter.

* Electronic address: lcouedel@mpe.mpg.de

[1] G. E. Morfill and A. V. Ivlev, Rev. Mod. Phys. 81, 1353 (2009).

[2] V. E. Fortov et al., Phys.-Usp. 47, 447 (2004); V. E. Fortov et al., Phys. Rep. 421, 1 (2005); P. K. Shukla and
B. Eliasson, Rev. Mod. Phys. 81, 25 (2009).

[3] S. Vladimirov, K. Ostrikov, and A. Samarian, Physics and Applications of Complex Plasmas (Imperial Press, London, 2005).

[4] H. M. Thomas and G. E. Morfill, Nature (London) 379, 806 (1996).

[5] C. H. Chiang and L. I, Phys. Rev. Lett. 77, 647 (1996).

[6] J. M. Kosterlitz and D. J. Thouless, J. Phys. C 6, 1181 (1973); B. I. Halperin and D. R. Nelson, Phys. Rev. Lett. 41, 121 (1978); D. R. Nelson and B. I. Halperin, Phys. Rev. B 19, 2457 (1979); A. P. Young, Phys. Rev. B 19, 1855 (1979).

[7] S. T. Chui, Phys. Rev. Lett. 48, 933 (1982); S. T. Chui, Phys. Rev. B 28, 178 (1983).

[8] C. A. Murray and D. H. Van Winkle, Phys. Rev. Lett. 58, 1200 (1987).

[9] K. Zahn and G. Maret, Phys. Rev. Lett. 85, 3656 (2000).

[10] R. A. Quinn et al., Phys. Rev. E 53, R2049 (1996).

[11] T. E. Sheridan, Phys. Plasmas 15, 103702 (2008).

[12] V. Nosenko et al., Phys. Rev. Lett. 103, 015001 (2009).

[13] For 2D plasma crystals, the "trivial" origin of the nonHamiltonial behavior associated with neutral gas friction can often be neglected, since the corresponding damping rate is typically one-to-two orders of magnitude smaller that the Einstein frequency [1].

[14] S. V. Vladimirov and M. Nambu, Phys. Rev. E 52, R2172 (1995).

[15] S. V. Vladimirov and O. Ishihara, Phys. Plasmas 3, 444 (1996).

[16] A. Melzer, V. A. Schweigert, and A. Piel, Phys. Rev. Lett. 83, 3194 (1999).

[17] The actio et reactio principle is only violated for microparticles; for "complete" complex plasmas, with the ambient ions and electrons taken into account, this principle is of course satisfied.

[18] T. Araki and H. Tanaka, Europhys. Lett. 82, 18004 (2008).

[19] T. Beatus, T. Tlusty, and R. Bar-Ziv, Nature Physics 2, 743 (2006).

[20] A. Melzer et al., Phys. Rev. E 54, R46 (1996).

[21] S. V. Vladimirov, P. V. Shevchenko, N. F. Cramer, Phys. Rev. E 56, R74 (1997).

[22] L. Couëdel et al., Phys. Rev. Lett. 103, 215001 (2009).

[23] A. V. Ivlev and G. Morfill, Phys. Rev. E 63, 016409 (2000).

[24] A. V. Ivlev et al., Phys. Rev. E 68, 026405 (2003).

[25] I. V. Schweigert et al., Phys. Rev. E 62, 1238 (2000).

[26] O. S. Vaulina et al., Phys. Rev. E 60, 5959 (1999).

[27] S. Nunomura et al., Phys. Rev. Lett. 83, 1970 (1999).

[28] V. Steinberg et al., Phys. Rev. Lett. 86, 4540 (2001).

[29] Changing either of these discharge parameters simultaneously affects all plasma characteristics (e.g., plasma density, particle charge, etc.). However, these parameters appear to be the most efficient in triggering the melting.

[30] See EPAPS Document No. XXXX for movie S1 and figure S2.

[31] S. Nunomura et al., Phys. Rev. Lett. 89, 035001 (2002).

[32] S. K. Zhdanov, A. V. Ivlev, and G. E. Morfill, Phys. Plasmas 16, 083706 (2009).

[33] Structure of the hybrid mode is also shown in Fig. 3 of Ref. 32]; note the difference between the reference frames used in [32] and in our paper.

[34] Here we leave aside the discussion of "localized" mechanisms of melting, e.g., due to defects. 